\title{
Dependence of the adsorption height of graphenelike adsorbates on their dimensionality
}

\author{
S. Weiß, ${ }^{1,2}$ D. Gerbert, ${ }^{3}$ A. Stein, ${ }^{3}$ A. K. Schenk,${ }^{4,}{ }^{*}$ X. Yang, ${ }^{1,2}$ C. Brülke, ${ }^{5}$ R. Kremring, ${ }^{5}$ S. Feldmann,${ }^{3, \dagger}$ F. C. Bocquet, ${ }^{1,2}$ \\ M. Gille, ${ }^{6}$ S. Hecht, ${ }^{6}$ M. Sokolowski, ${ }^{5}$ P. Tegeder, ${ }^{3}$ S. Soubatch, ${ }^{1,2, \ddagger}$ and F. S. Tautz ${ }^{1,2}$ \\ ${ }^{1}$ Peter Grünberg Institut (PGI-3), Forschungszentrum Jülich, 52425 Jülich, Germany \\ ${ }^{2}$ Jülich Aachen Research Alliance (JARA), Fundamentals of Future Information Technology, 52425 Jülich, Germany \\ ${ }^{3}$ Physikalisch-Chemisches Institut, Ruprecht-Karls-Universität Heidelberg, Im Neuenheimer Feld 253, 69120 Heidelberg, Germany \\ ${ }^{4}$ Department of Chemistry and Physics, La Trobe Institute for Molecular Sciences, La Trobe University, Victoria 3086, Australia \\ ${ }^{5}$ Institut für Physikalische und Theoretische Chemie der Universität Bonn, Wegelerstraße 12, 53115 Bonn, Germany \\ ${ }^{6}$ Department of Chemistry, Humboldt-Universität zu Berlin, Brook-Taylor-Straße 2, 12489 Berlin, Germany
}

(Received 21 December 2017; published 10 August 2018)

\begin{abstract}
Comparing the adsorption heights of various graphene nanoribbons on $\mathrm{Cu}(111)$ and $\mathrm{Au}(111)$ surfaces to those of graphene and $\pi$-conjugated planar organic molecules, we observe that two-dimensional graphene adsorbs much further away from the surface than both one-dimensional graphene nanoribbons and $\pi$-conjugated planar molecules - which represent zero-dimensional graphene flakes. We show that this is a direct consequence of the adsorbates' dimensionality. Our results provide invaluable insights into the interplay of Pauli repulsion, pushback effect, and chemical interaction for graphenelike adsorbates of any dimensionality on metal surfaces.
\end{abstract}

DOI: 10.1103/PhysRevB.98.075410

\section{INTRODUCTION}

Graphene nanoribbons (GNRs) attract attention because, unlike pristine graphene, they have a band gap [1-3] which makes them applicable in semiconductor technology. Because their electronic, magnetic, and transport properties depend on their width and edge shape [1,2,4], efforts are made to control their geometry. These attempts have resulted in techniques to produce atomically precise GNRs in solution and on surfaces [5-8]. An open question concerns the interaction of GNRs with their substrates. GNRs are $\pi$-conjugated objects, and as such similar to planar $\pi$-conjugated molecules on the one hand and graphene on the other. In fact, one might expect that the $s p^{2}$-hybridized carbon honeycomb lattice that is present in all of these adsorbates would interact chemically in a similar way with the metal substrate. As a secondary aspect, the edges of GNRs might form local bonds to the substrate, and thus the edge configuration could influence the interaction with the substrate.

It is well known that the adsorption height constitutes an important geometric parameter for the quantification of the interaction of an adsorbate with the substrate surface [9-11]. Therefore, we investigate here the adsorption height of various GNRs on metal substrates with the x-ray standing-wave technique [12]. We employ two noble-metal (111) surfaces of very different reactivities, $\mathrm{Au}$ and $\mathrm{Cu}$, and compare to $\pi$-conjugated molecules and graphene. We show that, regardless of their edge shape and their chemical edge structure (armchair or chiral vs

\footnotetext{
*Present address: Center for Quantum Spintronics, Department of Physics, Norwegian University of Science and Technology, NO-7491 Trondheim, Norway.

${ }^{\dagger}$ Present address: Cavendish Laboratory, University of Cambridge, J J Thomson Avenue, Cambridge CB3 OHE, United Kingdom.

${ }^{\ddagger}$ Corresponding author: s.subach@fz-juelich.de
}

chevron geometry, nitrogen-doped vs undoped edges), GNRs have a backbone adsorption height that is similar to that of $\pi$-conjugated planar molecules on the respective surfaces $[10,11,13,14]$, seemingly confirming that the interaction of the carbon honeycomb lattice with the metal determines the interaction strength and therefore also the adsorption height. In the light of this finding it is all the more surprising that graphene exhibits a very different adsorption height. As we will argue below, this is an immediate consequence of its dimensionality.

\section{EXPERIMENTAL DETAILS}

All experiments were conducted in ultrahigh vacuum at a base pressure below $10^{-9}$ mbar. As precursor molecules for the on-surface nanoribbon synthesis we employed 10,10'-dibromo-9,9'-bianthryl (DBBA) to synthesize the armchair 7-AGNR on $\mathrm{Au}(111)$ [5] and the chiral edge (3,1)GNR on $\mathrm{Cu}(111)$ [15], 6,11-dibromo-1,2,3,4-tetraphenyltriphenylene (DBTPTP) and 6,11-dibromo-1,4-diphenyl-2,3di(4'-pyridyl)-triphenylene (2N-DBTPTP) for the chevron CGNR [5] and the nitrogen-doped chevron NCGNR [16], respectively (Fig. 1). After depositing the precursors on the metal, the samples were annealed at $T_{\text {an }}=500-550^{\circ} \mathrm{C}$ to trigger polymerization and cyclodehydrogenation according to published recipes [5,15-19]. We also prepared $(3,1)$ $\mathrm{GNR} / \mathrm{Cu}(111)$ at lower annealing temperature $T_{\text {an }}$ to pinpoint the effect of edge dehydrogenation which is promoted by increasing temperature $[17,20]$. The formation of GNRs was confirmed by x-ray photoelectron spectroscopy (XPS) at offBragg conditions (cf. the Supplemental Material, Figs. 1 and $2[21])$.

Note that two different types of GNRs have been reported to form on $\mathrm{Cu}(111)$ from the DBBA precursor, 7-AGNR [17], and (3,1)-GNR [15]. Details have been discussed in the literature [15,17,22-27]. The difference between these 
(a)

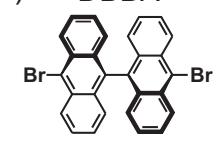

(b) DBTPTP

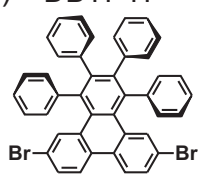

(c) 2N-DBTPTP

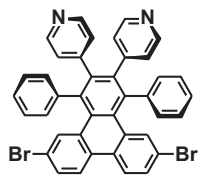

(d) 7-AGNR

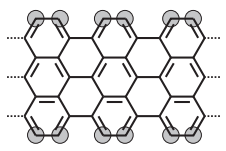

(e) (3,1)-GNR
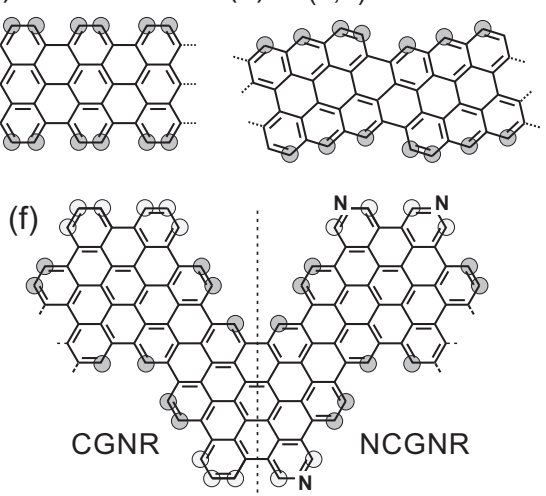

$-\ll \mathrm{C} 1 \propto \mathrm{C} 2 \propto<\mathrm{C} 3$

FIG. 1. Structure formulas of (a)-(c) the precursor molecules and (d)-(f) graphene nanoribbons. Note the nonplanar geometry of all precursor molecules. Different types of chemically inequivalent carbon atoms are marked: $\mathrm{C} 1$, atoms of the backbone; $\mathrm{C} 2$ and $\mathrm{C} 3$, different types of edge carbon atoms.

two types of GNRs is barely traceable in XPS, because both nanoribbons have the same ratio of the edge carbon atoms and the backbone carbon atoms $(\mathrm{C} 2 / \mathrm{C} 1)$ (cf. Fig. 1). In the present work, we assign the GNRs synthesized from DBBA to $(3,1)$-GNR, because our annealing temperature is close to that used in Ref. [15].

To grow graphene on $\mathrm{Cu}(111)$ we further annealed NCGNR/Cu(111) at $775^{\circ} \mathrm{C}$. The successful preparation of graphene was confirmed by the following observations: (1) Only one carbon component (C1) is present in the XPS spectrum [Fig. 2(c)], (2) low-energy electron diffraction (LEED) shows an arclike intensity close to the $\mathrm{Cu}(111)$ spots [cf. Supplemental Material, Fig. 3(a) [21]], and (3) angle-resolved photoelectron spectroscopy (ARPES) reveals a Dirac cone with the Dirac point at a binding energy of $0.4 \mathrm{eV}$ [cf. Supplemental Material, Fig. 3(b) [21]]. The quality of graphene is comparable to that reported in Ref. [28] as LEED and ARPES data show.

To determine the adsorption heights we applied the normal incidence $\mathrm{x}$-ray standing-wave (NIXSW) technique [12] which combines dynamical x-ray diffraction and photoelectron spectroscopy. NIXSW measurements were performed at the beamline I09 of the Diamond Light Source in Didcot, UK. In the NIXSW experiment, the $\mathrm{C} 1 s$ and $\mathrm{N} 1 s$ core-level spectra were recorded as a function of photon energy $h v$ close to the (111) Bragg condition of $\mathrm{Au}$ and $\mathrm{Cu}$ single crystals (2635 and $2970 \mathrm{eV}$, correspondingly). Photoelectron yield data were fitted with the program TORRICELLI [29], taking the angular resolution of the electron analyzer and angle-dependent nondipolar correction parameters [30-32] into account. The fitting models for XPS spectra that we used in our NIXSW analysis are shown in the Supplemental Material, Fig. 2 [21].

The NIXSW fits result in coherent positions $P_{\mathrm{c}}^{i}$ and coherent fractions $F_{\mathrm{c}}^{i}$ for each distinct species $i . P_{\mathrm{c}}^{i}$ is related to the average adsorption height with respect to the bulk-extended Bragg planes and $F_{\mathrm{c}}^{i}$ to the width of the height distribution.

The adsorption heights $h^{\text {abs }}$ for $\mathrm{Au}(111)$ are corrected for the herringbone surface reconstruction. This reconstruction
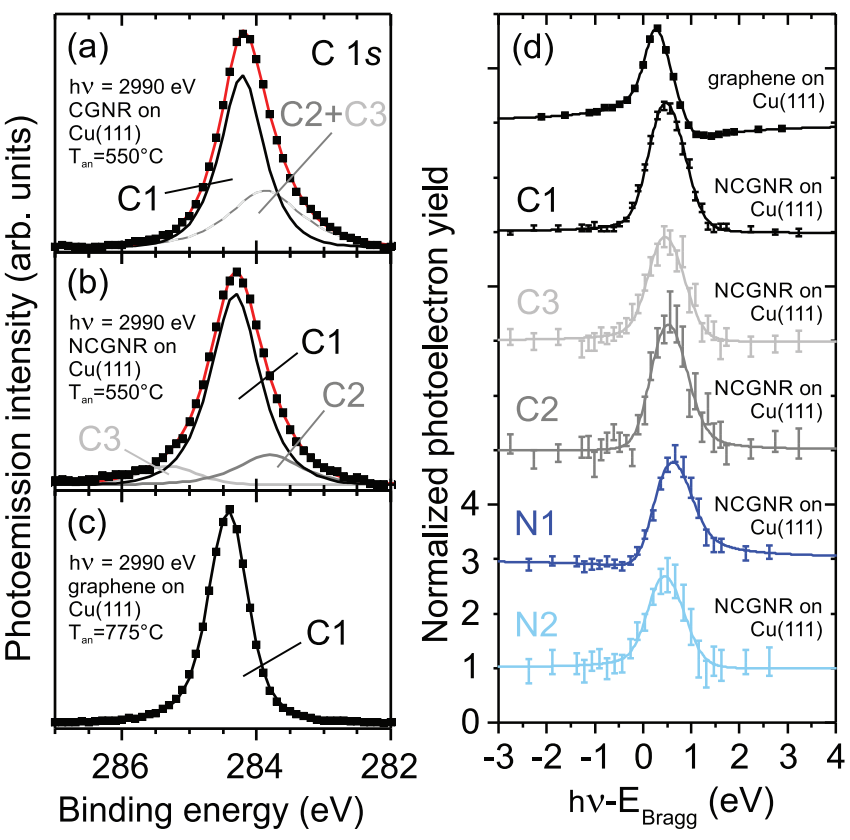

Binding energy (eV)
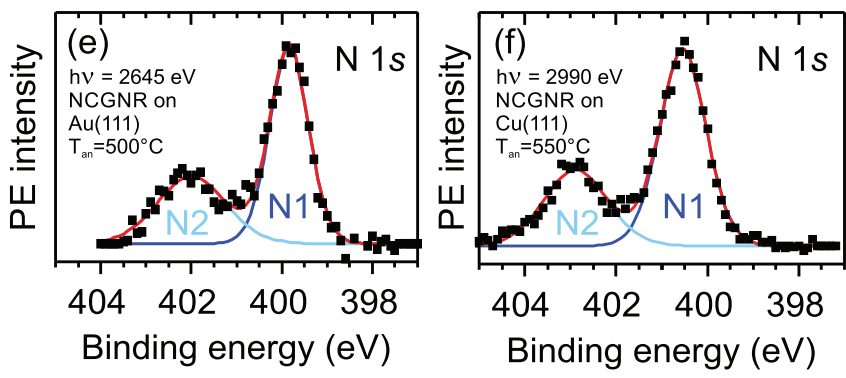

FIG. 2. (a)-(c) Experimental C $1 s$ XPS spectra measured in offBragg condition at photon energy $h v=2990 \mathrm{eV}$ (symbols) and fitting models (solid lines) for (a) CGNR nanoribbons, (b) NCGNR nanoribbons, and (c) graphene on $\mathrm{Cu}(111)$. (d) Experimental photoelectron yield curves (symbols and error bars) and fits (solid lines) for graphene and various carbon and nitrogen species of NCGNR/Cu(111). (e),(f) Off-Bragg N $1 s$ XPS spectra for NCGNRs on (e) Au(111) and (f) $\mathrm{Cu}(111)$.

leads to a sinusoidal buckling of the topmost Au layer with a vertical amplitude of approximately $0.15 \AA$ [33] and an averaged outward relaxation of the topmost layer by $0.071 \AA$ [34]. The $\mathrm{Cu}(111)$ contraction due to the surface relaxation [35] is below the NIXSW uncertainty and was therefore neglected. The van der Waals ( $\mathrm{vdW}$ ) radii used to transform absolute adsorption heights $h^{\text {abs }}$ into vdW-reduced adsorption heights $h^{\mathrm{vdW}}$ are listed in the Supplemental Material, Table 1 [21].

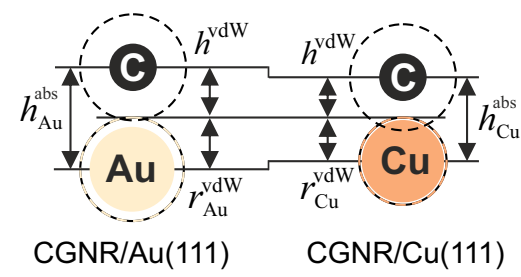

FIG. 3. Illustration of the $h^{\mathrm{vdW}}$ definition. Dashed circles are van der Waals spheres with radii $r_{\mathrm{Au}}^{\mathrm{vdW}}, r_{\mathrm{Cu}}^{\mathrm{vdW}}$, and $r_{\mathrm{C}}^{\mathrm{vdW}}$. For simplicity, carbon atoms are drawn in on-top positions. 


\section{RESULTS}

\section{A. Chemical composition of the graphene nanoribbons}

After on-surface GNR synthesis, we carried out x-ray photoemission spectroscopy with a photon energy a few tens of eV above the Bragg energy. This serves two purposes. On the one hand, the XPS spectra confirm the successful synthesis of the desired GNR, and on the other hand, the analysis provides fitting models for the subsequent standing-wave experiments with photon energies around the Bragg energy.

Figures 2(a)-2(c) show the off-Bragg C $1 s$ spectra of CGNR and NCGNR nanoribbons and graphene on $\mathrm{Cu}(111)$. Note that only exemplary data are displayed (cf. Supplemental Material, Fig. 2 [21], for data on other GNRs). The C $1 s$ spectra of CGNRs, and also 7-AGNR and $(3,1)-G N R$, consist of two peaks separated by approximately $0.4 \mathrm{eV}$. The peak at $284.3 \mathrm{eV}$ in Fig. 2(a) is assigned to $s p^{2}$-hybridized C atoms with bonds to three other $\mathrm{C}$ atoms [carbon backbone, labeled $\mathrm{C} 1$ in Figs. 1(d)-1(f)] and the less intense peak at smaller binding energy $(283.9 \mathrm{eV})$ to the atoms at the GNR edge [labeled C2 in Figs. 1(d)-1(f)], in agreement with Ref. [17]. We stress that the $\mathrm{C} 2$ component may include contributions from atoms in dissimilar states. In particular, one may expect differences in local adsorption configurations of individual atoms and the dehydrogenation of some of the edge atoms due to the high-temperature treatment $[17,20]$. For the chevron nanoribbons (CGNRs and NCGNRs) we moreover separate edge atoms of types C2 and C3 [Fig. 1(f)]. In the case of NCGNRs these two species can be spectroscopically differentiated due to a chemical shift caused by nitrogen atoms in the immediate environment [Fig. 2(b)]. The intensity ratios C2/C1 for 7-AGNR, (3,1)-GNR and CGNRs and C3/C2/C1 for NCGNRs agree well with the stoichiometry of the corresponding nanoribbons. The spectrum of graphene includes only the $\mathrm{C} 1$ component [Fig. 2(c)].

The $\mathrm{N} 1 s$ spectra of NCGNRs on $\mathrm{Au}(111)$ and $\mathrm{Cu}(111)$ consist of two components N1 and N2 [Figs. 2(e) and 2(f)]. It is known that NCGNRs tend to cluster on $\mathrm{Au}(111)$ [36], caused by hydrogen bonding of the $\mathrm{N}$ atoms to neighboring GNRs [7]. We assign the N2 component to atoms involved in this interribbon bonding, while $\mathrm{N} 1$ atoms are ascribed to atoms that do not take part in this interaction. The large chemical shift is a consequence of different adsorption heights for these two species (see below). It is well known that intermolecular interactions can cause substantial height differences [37], and such height differences in turn may cause substantial chemical shifts [38]. The splitting of N1 and N2 of NCGNRs on $\mathrm{Cu}(111)$ [Fig. 2(f)] suggests that NCGNRs tend to cluster on $\mathrm{Cu}(111)$ as well. Because at the junction between two NCGNRs only half of the $\mathrm{N}$ atoms are involved in bonding [36], the expected $\mathrm{N} 2 /(\mathrm{N} 1+\mathrm{N} 2)$ intensity ratio ranges from 0 (no interribbon bonding) to 0.5 (all ribbons bond). The observed N2/(N1 + $\mathrm{N} 2)$ ratios of $0.38(\mathrm{Au})$ and $0.35(\mathrm{Cu})$ are in this range.

\section{B. Adsorption heights of the graphene nanoribbons}

To measure the adsorption height of nanoribbons and graphene we employed NIXSW technique, which provides the adsorption heights of chemical species relative to the Bragg planes of the substrate crystal, extended from its bulk to the

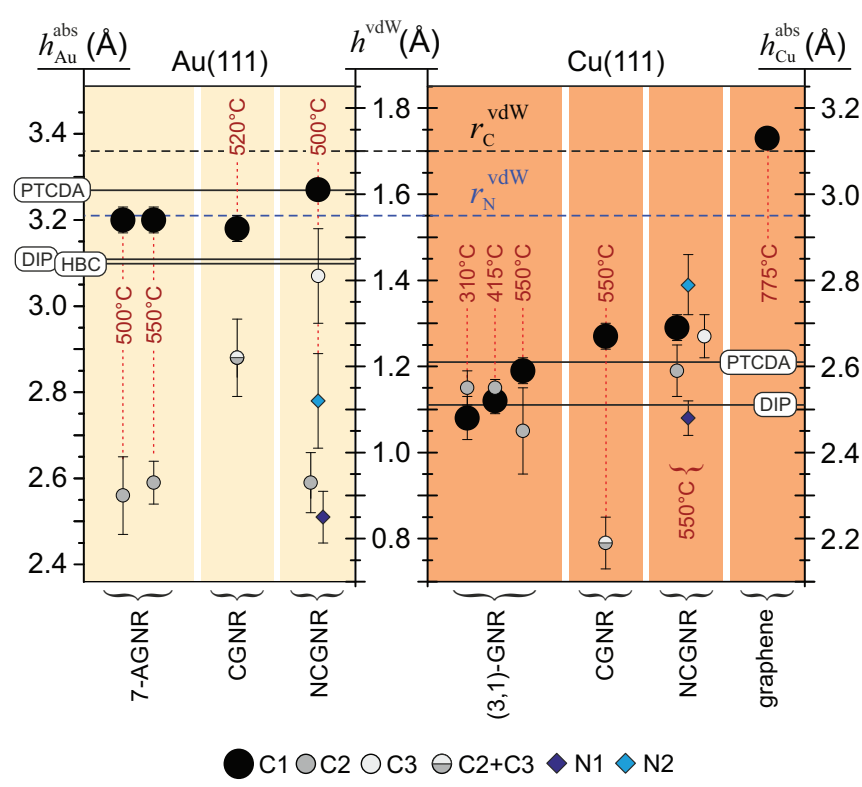

FIG. 4. Absolute adsorption heights $h_{\mathrm{Au}}^{\mathrm{abs}}$ for various species, as indicated by the symbols, on $\mathrm{Au}(111)$ (left panel) and $h_{\mathrm{Cu}}^{\mathrm{abs}}$ on $\mathrm{Cu}(111)$ (right panel), as well as vdW-reduced heights $h^{\mathrm{vdW}}$ (central axis) for different GNRs and graphene. The dashed lines denote van der Waals radii of carbon $r_{\mathrm{C}}^{\mathrm{vdW}}$ (black) and nitrogen $r_{\mathrm{N}}^{\mathrm{vdW}}$ (blue) [40]. The solid black lines show adsorption heights of perylenetetracarboxylic dianhydride (PTCDA) [10,11], diindenoperylene (DIP) [13], and hexa-peri-hexabenzocoronene (HBC) [14]. Numbers in red specify annealing temperature $T_{\text {an }}$.

near-surface area [12]. In the following discussion we use both absolute adsorption heights $h^{\text {abs }}$ and vdW-reduced adsorption heights $h^{\mathrm{vdW}}$, defined as the heights above the van der Waals spheres of the substrate atoms [39]. The two are related by $h^{\mathrm{vdW}}=h^{\mathrm{abs}}-r_{\text {substrate }}^{\mathrm{vdW}}$, with $r_{\mathrm{Au}}^{\mathrm{vdW}}=1.66 \AA$ and $r_{\mathrm{Cu}}^{\mathrm{vdW}}=$ $1.40 \AA$ [40]. A graphical representation of $h^{\mathrm{vdW}}$ and $h^{\mathrm{abs}}$ is given in Fig. 3. vdW-reduced adsorption heights have proven to be a valuable quantity to rationalize the adsorption of organic molecules on surfaces and, in particular, to demonstrate the differences in adsorption mechanisms at surfaces of different chemical reactivity $[32,39,41]$.

Figure 4 shows $h^{\text {abs }}$ and $h^{\mathrm{vdW}}$ for the various components of different nanoribbons on $\mathrm{Au}(111)$ and $\mathrm{Cu}(111)$. Corresponding coherent positions $P_{\mathrm{c}}^{i}$ and coherent fractions $F_{\mathrm{c}}^{i}$ are listed in Table I. A first important observation is that both $h^{\text {abs }}$ and $h^{\mathrm{vdW}}$ of the GNR backbone (C1) strongly depend on the substrate but barely on the type of GNR, although on $\mathrm{Cu}(111)$ the spread between the various GNRs is slightly larger. On $\mathrm{Au}(111)$, with $h_{\mathrm{C} 1}^{\mathrm{abs}} \simeq 3.2 \AA, h_{\mathrm{C} 1}^{\mathrm{vdW}}$ is only $0.15 \AA$ below the vdW radius $r_{\mathrm{C}}^{\mathrm{vdW}}$ of carbon atoms. In agreement with Refs. [19,42-44], this indicates a physisorptive interaction of the $\mathrm{C} 1$ backbone with the metal. Thus, the GNRs essentially float on the spill-out electron density outside the surface, whose cutoff is approximately given by $r_{\mathrm{Au}}^{\mathrm{vdW}}$ [39], hence $h_{\mathrm{C} 1}^{\mathrm{vdW}} \approx r_{\mathrm{C}}^{\mathrm{vdW}}$ [41]. On $\mathrm{Cu}(111)$, where $h_{\mathrm{C} 1}^{\mathrm{abs}} \simeq 2.65 \AA, h_{\mathrm{C} 1}^{\mathrm{vd}}$ is $0.55 \AA$ smaller than $r_{\mathrm{C}}^{\mathrm{vdW}}$ and the nanoribbons thus penetrate the electron density at the surface, which points to a chemisorptive interaction of the GNR backbone with the metal [41].

The high coherent fractions $F_{\mathrm{c}}$ of $\mathrm{C} 1$ atoms found in all NIXSW measurements ( $>0.8$, cf. Table I) prove a high 
TABLE I. Coherent positions $P_{c}$, coherent fractions $F_{c}$, and absolute adsorption heights $h^{\text {abs }}$ of carbon and nitrogen atoms for 7$\operatorname{AGNR}\left(T_{\text {an }}=550^{\circ} \mathrm{C}\right), \operatorname{CGNRs}\left(T_{\text {an }}=520^{\circ} \mathrm{C}\right)$, and $\operatorname{NCGNRs}\left(T_{\text {an }}=\right.$ $\left.500^{\circ} \mathrm{C}\right)$ on $\mathrm{Au}(111)$ and $(3,1)-\mathrm{GNR}\left(T_{\text {an }}=550^{\circ} \mathrm{C}\right)$, CGNRs $\left(T_{\text {an }}=\right.$ $\left.550^{\circ} \mathrm{C}\right)$, NCGNRs $\left(T_{\text {an }}=550^{\circ} \mathrm{C}\right)$, and graphene $\left(T_{\text {an }}=775^{\circ} \mathrm{C}\right)$ on $\mathrm{Cu}(111)$. The $F_{c}$ exceeding unity in case of $\mathrm{C} 1$ components of $(3,1)$ $\mathrm{GNR} / \mathrm{Cu}(111), \mathrm{CGNR} / \mathrm{Cu}(111)$, and $\mathrm{NCGNR} / \mathrm{Cu}(111)$ are due to technical issues caused by the wide angular acceptance of the electron analyzer [31] and a possible nonlinear response of its microchannel plate detector [45]. These issues are known to have only a negligible effect on the coherent positions. The relatively low $F_{\mathrm{c}}$ for graphene can be attributed to a possible vertical corrugation (cf., e.g., Ref. [46]) or to structural defects of graphene grown from GNR precursors at a much smaller temperature than the temperature usually used for graphene synthesis [47].

\begin{tabular}{|c|c|c|c|c|}
\hline & & $P_{\mathrm{c}}$ & $F_{\mathrm{c}}$ & $h^{\text {abs }}(\AA)$ \\
\hline \multicolumn{5}{|c|}{$\mathrm{Au}(111)$} \\
\hline \multirow[t]{2}{*}{ 7-AGNR } & $\mathrm{C} 1$ & 0.39 & 0.89 & $3.20 \pm 0.03$ \\
\hline & $\mathrm{C} 2$ & 0.12 & 0.46 & $2.59 \pm 0.05$ \\
\hline \multirow[t]{2}{*}{ CGNR } & $\mathrm{C} 1$ & 0.38 & 0.98 & $3.18 \pm 0.03$ \\
\hline & $\mathrm{C} 2$ & 0.25 & 0.40 & $2.88 \pm 0.09$ \\
\hline \multirow[t]{6}{*}{ NCGNR } & $\mathrm{C} 1$ & 0.42 & 0.82 & $3.27 \pm 0.02$ \\
\hline & $\mathrm{C} 2$ & 0.13 & 0.39 & $2.59 \pm 0.07$ \\
\hline & $\mathrm{C} 3$ & 0.33 & 0.15 & $3.07 \pm 0.11$ \\
\hline & N1 & 0.10 & 0.18 & $2.51 \pm 0.06$ \\
\hline & N2 & 0.21 & 0.40 & $2.78 \pm 0.11$ \\
\hline & & \multicolumn{3}{|c|}{$\mathrm{Cu}(111)$} \\
\hline \multirow[t]{2}{*}{$(3,1)-G N R$} & $\mathrm{C} 1$ & 0.24 & 1.13 & $2.59 \pm 0.03$ \\
\hline & $\mathrm{C} 2$ & 0.18 & 0.37 & $2.45 \pm 0.10$ \\
\hline \multirow[t]{2}{*}{ CGNR } & $\mathrm{C} 1$ & 0.27 & 1.21 & $2.67 \pm 0.02$ \\
\hline & $\mathrm{C} 2$ & 0.04 & 0.95 & $2.19 \pm 0.06$ \\
\hline \multirow[t]{5}{*}{ NCGNR } & $\mathrm{C} 1$ & 0.28 & 1.04 & $2.69 \pm 0.03$ \\
\hline & $\mathrm{C} 2$ & 0.23 & 0.90 & $2.59 \pm 0.06$ \\
\hline & $\mathrm{C} 3$ & 0.27 & 0.80 & $2.67 \pm 0.05$ \\
\hline & N1 & 0.18 & 0.61 & $2.48 \pm 0.04$ \\
\hline & $\mathrm{N} 2$ & 0.33 & 0.49 & $2.79 \pm 0.07$ \\
\hline Graphene & $\mathrm{C} 1$ & 0.49 & 0.61 & $3.13 \pm 0.02$ \\
\hline
\end{tabular}

degree of vertical order. Importantly, the adsorption heights of the GNR backbones are similar to those of graphenelike planar molecules such as perylenetetracarboxylic dianhydride (PTCDA), diindenoperylene (DIP), and hexa-perihexabenzocoronene (HBC) (see Fig. 4), both on $\mathrm{Au}(111)$ and $\mathrm{Cu}(111)$ [10,11,13,14], suggesting a similar adsorption mechanism [41] for these molecules and GNRs. In particular, the especially strong attractive chemical interaction of the honeycomb lattice in the GNR backbone with $\mathrm{Cu}$, evidenced by $h_{\mathrm{C} 1}^{\mathrm{vdW}}<r_{\mathrm{C}}^{\mathrm{vdW}}$, is also found for molecules [32].

\section{Internal distortions of adsorbed nanoribbons}

We now discuss the distortion of the GNRs upon adsorption as evidenced by the height differences between their backbones (C1 atoms) and edge atoms ( $22, \mathrm{~N} 1$, and N2). The distortion reveals further details of the GNR-substrate interaction. On $\mathrm{Au}(111)$, the edge carbons $\mathrm{C} 2$ of 7-AGNR and NCGNRs are $\simeq 0.7 \AA$ below the carbon backbone atoms $\mathrm{C} 1$. This is a massive distortion that suggests substantial edge dehydrogenation, which at our annealing temperatures (see Fig. 4) is indeed expected [20], followed by the formation of local covalent bonds between the $\mathrm{C} 2$ atoms and the $\mathrm{Au}(111)$ substrate.

Remarkably, with $h_{\mathrm{C} 2}^{\mathrm{abs}} \simeq 2.6 \AA$ the $\mathrm{C} 2$ adsorption heights for 7-AGNR/Au(111), NCGNR/Au(111), (3,1)-GNR/Cu(111), and NCGNR/Cu(111) are almost identical, i.e., nearly independent of the type of nanoribbon and even the surface. This suggests similar bonding configurations of the C2 atoms in these cases. The lateral position at which the least dependence of the adsorption height on the substrate and specifically its $r_{\text {substrate }}^{\mathrm{vdW}}$ is expected is the hollow site between three substrate atoms. We therefore conclude that carbon edge atoms C2 preferentially sit in hollow sites of the (111) surfaces from where they form local bonds with $\mathrm{Au}$ and $\mathrm{Cu}$. Note, however, that this local C2-metal bond has little influence on the adsorption height of the $\mathrm{C} 1$ backbone. On $\mathrm{Au}(111)$, in particular, Pauli repulsion from the metal keeps the $\mathrm{C} 1$ atoms far above the $\mathrm{C} 2$ atoms. In contrast, on $\mathrm{Cu}(111)$, where also the backbone $\mathrm{C} 1$ interacts chemically with the substrate, the $\mathrm{C} 2$ vs $\mathrm{C} 1$ distortion is hence much smaller.

For NCGNRs, the N1 atoms on both surfaces are found at similar adsorption heights as the carbon edge atoms $\mathrm{C} 2$ ( $h_{\mathrm{C} 2}^{\mathrm{abs}} \simeq h_{\mathrm{N} 1}^{\mathrm{abs}} \simeq 2.5 \AA$ ), and moreover the variation of $h_{\mathrm{N} 1}^{\mathrm{abs}}$ between $\mathrm{Cu}(111)$ and $\mathrm{Au}(111)$ is small. This suggests that $\mathrm{N} 1$ atoms also form local bonds to the metal, with a common bonding configuration on the two surfaces. The N2 atoms are found $\simeq 0.3 \AA$ above the $\mathrm{N} 1$ atoms. This is consistent with their assignment to $\mathrm{N}$ atoms that take part in interribbon bonding, as this weakens the interaction with the substrate and lifts them up [37].

According to Fig. 1(f), in the case of NCGNRs we also need to distinguish between standard carbon edge atoms $\mathrm{C} 2$ and carbon edge atoms in the vicinity of $\mathrm{N}$ atoms (C3). On both $\mathrm{Au}(111)$ and $\mathrm{Cu}(111)$, the $\mathrm{C} 3$ atoms have an adsorption height that is larger than that of the $\mathrm{C} 2$ atoms. This is a consequence of their attachment to nitrogens, some of which are lifted due to interribbon bonding. In line with the larger overall $\mathrm{C} 1$ vs $\mathrm{C} 2$ distortion on $\mathrm{Au}(111)$, the distance between $\mathrm{C} 2$ and $\mathrm{C} 3$ is $\simeq 0.5 \AA$ for $\mathrm{Au}(111)$, but only $\simeq 0.1 \AA$ for $\mathrm{Cu}(111)$. The low coherent fraction $F_{\mathrm{c}}=0.15$ of $\mathrm{C} 3$ on $\mathrm{Au}(111)$ is caused by the fact that not all nitrogen atoms are at the same height.

Edge atoms for CGNRs merit a separate discussion. Because CGNRs on $\mathrm{Au}(111)$ tend to cluster [48], the adsorption heights of $\mathrm{C} 2$ and $\mathrm{C} 3$ edge atoms will differ if interribbon contacts that only affect C3 are present. However, for CGNRs we cannot differentiate between $\mathrm{C} 2$ and $\mathrm{C} 3$ in x-ray photoemission spectroscopy [Fig. 2(a)], in contrast to the situation for NCGNRs where nitrogen serves as a chemical marker to distinguish $\mathrm{C} 2$ atoms from the $\mathrm{N}$-coordinated $\mathrm{C} 3$ atoms. Hence, for CGNRs the NIXSW analysis yields an average adsorption height for $\mathrm{C} 2$ and $\mathrm{C} 3$. The observed value of $h_{\mathrm{C} 2 / \mathrm{C} 3}^{\mathrm{abs}}=2.9 \AA$ in Fig. 4 can be qualitatively explained by a $\mathrm{C} 2$ adsorption height close to that of other nanoribbons ( $h_{\mathrm{C} 2}^{\mathrm{abs}} \simeq 2.6 \AA$ as for NCGNRs) and a C3 adsorption height that is increased by lateral interactions to $h_{\mathrm{C} 3}^{\mathrm{abs}} \simeq 3.0 \AA$. On $\mathrm{Cu}(111)$, the edge atoms of CGNRs are strongly attracted to the metal (Fig. 4). This is consistent with a weaker tendency to form lateral contacts to neighboring nanoribbons (cf. Ref. [48]), resulting in low heights of both $\mathrm{C} 2$ and $\mathrm{C} 3$.

We note that the vertical coherence of all atoms $(\mathrm{C} 1, \mathrm{C} 2, \mathrm{C} 3$, $\mathrm{N} 1$, and N2) for all GNRs on $\mathrm{Cu}(111)$ is found to be noticeably 
higher than on $\mathrm{Au}(111)$, where the herringbone reconstruction reduces the coherence.

The effect of the annealing temperature $T_{\mathrm{an}}$, illustrated in Fig. 4 for the example of $(3,1)-G N R / C u(111)$, reveals further aspects of the substrate bonding mechanism. Raising $T_{\text {an }}$ from 310 to $550{ }^{\circ} \mathrm{C}$ results in an increasing degree of dehydrogenation and lower adsorption heights of edge carbon atoms $\mathrm{C} 2$. At the same time, the adsorption height of the backbone $\mathrm{C} 1$ increases by approximately $0.1 \AA$. Note that the comparison of CGNRs to 7-AGNR on $\mathrm{Au}(111)$, as well as CGNRs to $(3,1)-G N R$ on $\mathrm{Cu}(111)$, fits into this trend of anticorrelated $\mathrm{C} 1 \mathrm{vs} \mathrm{C} 2 / \mathrm{C} 3$ adsorption heights, suggesting that the mechanism that underlies this anticorrelation (see below) is of a general nature and does not only apply to the $T_{\text {an }}$ series recorded for $(3,1)-\mathrm{GNR} / \mathrm{Cu}(111)$.

\section{DISCUSSION}

Summarizing our findings up to this point, we reach two main conclusions: First, as evidenced by substantial internal distortions, GNR edges react with both $\mathrm{Au}(111)$ and $\mathrm{Cu}(111)$, producing some small variations also of the adsorption height of the GNR backbone. Second, however, the latter is predominantly controlled by the interaction of carbon honeycomb lattice with the electron density in front of the metal surface, as revealed by the similarity with molecular adsorption heights for which this mechanism is well established [41].

In the light of these findings we now turn to graphene. The adsorption height of graphene on $\mathrm{Cu}(111)$ is measured to be $3.13 \AA$ (Fig. 4), corresponding to a $h^{\mathrm{vdW}}$ that is even $0.03 \AA$ larger than $r_{\mathrm{C}}^{\mathrm{vdW}}$, in agreement with theory [49-51]. This raises the question of why the two-dimensional lattice of graphene on $\mathrm{Cu}(111)$ does have a much larger adsorption height than graphenelike planar molecules and GNRs on the same surface. After all, the honeycomb lattice of the backbone which, as we have seen, determines the adsorption height of GNRs essentially irrespective of vertical distortions at the edges of the GNRs is the same in all three cases-molecules, GNRs, and graphene.

We suggest that the observed effect is a direct result of dimensionality and can be explained by a simple model that describes the interaction between the carbon honeycomb lattice and the substrate by a combination of Pauli repulsion, pushback effect, and chemical interaction [32,39,41]. The pushback effect [52] holds the key, because in its way of acting there is a crucial difference between zero-dimensional (0D) and one-dimensional (1D) adsorbates on the one hand and two-dimensional (2D) adsorbates on the other. In the case of planar molecules (OD) and GNRs (1D) the charge pushed back beneath the adsorbate can partially escape sideways around the adsorbate, allowing the adsorbate to sink into the spill-out charge density ["cushion effect," cf. Fig. 5(a)] and establish a chemical interaction with the substrate. In contrast, because 2D graphene is extended in both lateral directions, the charge cannot escape sideways but is pressed, for symmetry reasons, perpendicularly into the bulk. This is energetically unfavorable, with the result that graphene experiences an additional repulsion, preventing a chemical interaction even in the case of the reactive $\mathrm{Cu}(111)$ surface. Generally speaking, GNRs therefore do not constitute a good model system for the bonding (a)
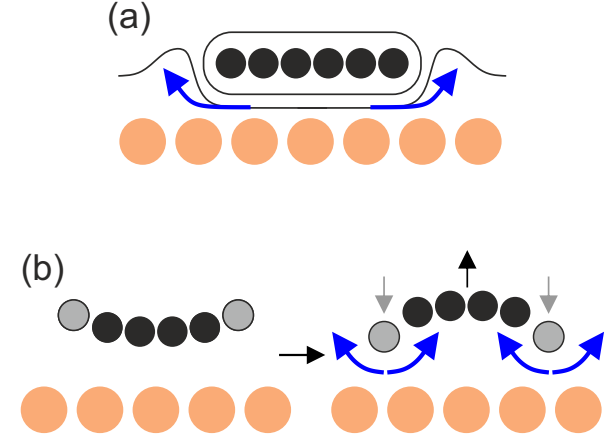

FIG. 5. Charge redistributions at the nanoribbon/metal interface. (a) Schematic illustration of the spill-out charge redistribution (blue arrows) for adsorption of planar molecules and graphene nanoribbons. (b) Schematic illustration of the annealing-induced change of the $(3,1)-\mathrm{GNR} / \mathrm{Cu}(111)$ geometry.

of an extended graphene sheet to a metal surface. Although their internal electronic delocalization is graphenelike in one direction, their finite width in the other direction adds a decisive aspect that enables them to bind to a metal surface significantly more strongly.

The validity of this interaction model for graphenelike adsorbates is confirmed by several additional observations. First, the model allows us to understand the anticorrelation of the $\mathrm{C} 1$ and $\mathrm{C} 2 / \mathrm{C} 3$ adsorption heights that we have mentioned above. When the $\mathrm{C} 2 / \mathrm{C} 3$ edge atoms move towards the substrate, they displace charge, which in turn pushes up the backbone $\mathrm{C} 1$ [Fig. 5(b)]. Second, the fact that among all GNRs reported in Fig. 4 the smallest $h^{\text {vdW }}$ on $\mathrm{Cu}(111)$ is found for $(3,1)$-GNR, i.e., the one having the most narrow footprint, is naturally explained by this interaction model, because the narrower the GNR the easier is the redistribution of the spill-out charge density from underneath the GNR. Interestingly, an analogous observation holds true for graphenelike planar molecules: the narrow pentacene molecule shows a smaller adsorption height on $\mathrm{Cu}(111)$ [53] than perylene derivatives $[11,13]$.

\section{CONCLUSIONS}

A systematic study of the adsorption heights of graphene nanoribbons on close-packed noble-metal surfaces and the comparison of these data with other adsorbates exhibiting a carbon honeycomb lattice has revealed the importance of the adsorbate's dimensionality for its adsorption height. The influence of dimensionality in turn is a direct consequence of the pushback and cushion effects at metal surfaces. Our observations suggest that before planar adsorbates of the type studied here can engage in chemical interaction with the (close-packed) metal substrate, they have to overcome Pauli repulsion by displacing some of the spill-out charge of the metal. This allows them to come sufficiently close to the surface to establish chemical bonding, including, e.g., wavefunction hybridization and charge transfer. If this displacement is impossible, for instance due to the dimensionality of the adsorbate, then Pauli repulsion has the capacity to suppress chemical interactions between adsorbate and substrate. The interaction model developed here for the case of graphenelike planar adsorbates appears to be applicable to other 2D materials 
[54] as well and is therefore of a more general nature. It thus offers important insights into the interaction mechanisms at metal surfaces, in particular the balance between repulsive and attractive interactions.

\section{ACKNOWLEDGMENTS}

We thank Diamond Light Source for access to beamline I09 (Proposals No. SI12627 and No. SI16056) that contributed to the results presented here. We thank D. A. Duncan, P.
K. Thakur, and T.-L. Lee for experimental support. S.W., S.S., and F.S.T. thank P. Jakob, Universität Marburg, for fruitful discussions. D.G., A.S., and P.T. acknowledge funding by the Deutsche Forschungsgemeinschaft through Project No. TE479/3-1 and the SFB 1249 "N-Heteropolyzyklen als Funktionsmaterialien” (Project No. B 06). F.C.B. and F.S.T. acknowledge funding by the Deutsche Forschungsgemeinschaft through the SFB 1083 "Structure and Dynamics of Internal Interfaces" (Project No. A 12). M.S. thanks the Deutsche Forschungsgemeinschaft for funding under Project No. SO407/6-3.
[1] K. Nakada, M. Fujita, G. Dresselhaus, and M. S. Dresselhaus, Edge state in graphene ribbons: Nanometer size effect and edge shape dependence, Phys. Rev. B 54, 17954 (1996).

[2] M. Y. Han, B. Özyilmaz, Y. Zhang, and P. Kim, Energy BandGap Engineering of Graphene Nanoribbons, Phys. Rev. Lett. 98, 206805 (2007).

[3] X. Li, X. Wang, L. Zhang, S. Lee, and H. Dai, Chemically derived, ultrasmooth graphene nanoribbon semiconductors, Science 319, 1229 (2008).

[4] K. Wakabayashi, M. Fujita, H. Ajiki, and M. Sigrist, Electronic and magnetic properties of nanographite ribbons, Phys. Rev. B 59, 8271 (1999).

[5] J. Cai, P. Ruffieux, R. Jaafar, M. Bieri, T. Braun, S. Blankenburg, M. Muoth, A. P. Seitsonen, M. Saleh, X. Feng, K. Müllen, and R. Fasel, Atomically precise bottom-up fabrication of graphene nanoribbons, Nature (London) 466, 470 (2010).

[6] A. Narita, X. Feng, Y. Hernandez, S. A. Jensen, M. Bonn, H. Yang, I. A. Verzhbitskiy, C. Casiraghi, M. R. Hansen, A. H. R. Koch, G. Fytas, O. Ivasenko, B. Li, K. S. Mali, T. Balandina, S. Mahesh, S. De Feyter, and K. Müllen, Synthesis of structurally well-defined and liquid-phase-processable graphene nanoribbons, Nat. Chem. 6, 126 (2014).

[7] A. Narita, X.-Y. Wang, X. Feng, and K. Müllen, New advances in nanographene chemistry, Chem. Soc. Rev. 44, 6616 (2015).

[8] P. Ruffieux, S. Wang, B. Yang, C. Sánchez-Sánchez, J. Liu, T. Dienel, L. Talirz, P. Shinde, C. A. Pignedoli, D. Passerone, T. Dumslaff, X. Feng, K. Müllen, and R. Fasel, On-surface synthesis of graphene nanoribbons with zigzag edge topology, Nature (London) 531, 489 (2016).

[9] A. Hauschild, K. Karki, B. C. C. Cowie, M. Rohlfing, F. S. Tautz, and M. Sokolowski, Molecular Distortions and Chemical Bonding of A Large $\pi$-Conjugated Molecule On a Metal Surface, Phys. Rev. Lett. 94, 036106 (2005).

[10] S. K. M. Henze, O. Bauer, T.-L. Lee, M. Sokolowski, and F. S. Tautz, Vertical bonding distances of PTCDA on $\mathrm{Au}(111)$ and $\operatorname{Ag}(111)$ : Relation to the bonding type, Surf. Sci. 601, 1566 (2007).

[11] A. Gerlach, S. Sellner, F. Schreiber, N. Koch, and J. Zegenhagen, Substrate-dependent bonding distances of PTCDA: A comparative $\mathrm{x}$-ray standing-wave study on $\mathrm{Cu}(111)$ and $\mathrm{Ag}(111)$, Phys. Rev. B 75, 045401 (2007).

[12] J. Zegenhagen, Surface structure determination with x-ray standing waves, Surf. Sci. Rep. 18, 199 (1993).
[13] C. Bürker, N. Ferri, A. Tkatchenko, A. Gerlach, J. Niederhausen, T. Hosokai, S. Duhm, J. Zegenhagen, N. Koch, and F. Schreiber, Exploring the bonding of large hydrocarbons on noble metals: Diindoperylene on $\mathrm{Cu}(111), \mathrm{Ag}(111)$, and $\mathrm{Au}(111)$, Phys. Rev. B 87, 165443 (2013).

[14] C. Bürker, Adsorption geometry of $\pi$-conjugated organic molecules on metal surfaces studied with the x-ray standing wave technique, Ph.D. thesis, Eberhard Karls Universität Tübingen, 2014.

[15] P. Han, K. Akagi, F. F. Canova, H. Mutoh, S. Shiraki, K. Iwaya, P. S. Weiss, N. Asao, and T. Hitosugi, Bottom-up graphenenanoribbon fabrication reveals chiral edges and enantioselectivity, ACS Nano 8, 9181 (2014).

[16] C. Bronner, S. Stremlau, M. Gille, F. Brauße, A. Haase, S. Hecht, and $\mathrm{P}$. Tegeder, Aligning the band gap of graphene nanoribbons by monomer doping, Angew. Chem. Int. Ed. 52, 4422 (2013).

[17] K. A. Simonov, N. A. Vinogradov, A. S. Vinogradov, A. V. Generalov, E. M. Zagrebina, N. Mårtensson, A. A. Cafolla, T. Carpy, J. P. Cunniffe, and A. B. Preobrajenski, Effect of substrate chemistry on the bottom-up fabrication of graphene nanoribbons: Combined core-level spectroscopy and STM study, J. Phys. Chem. C 118, 12532 (2014).

[18] C. Bronner, J. Björk, and P. Tegeder, Tracking and removing $\mathrm{Br}$ during the on-surface synthesis of a graphene nanoribbon, $\mathrm{J}$. Phys. Chem. C 119, 486 (2015).

[19] F. Maaß, M. Utecht, S. Stremlau, M. Gille, J. Schwarz, S. Hecht, T. Klamroth, and P. Tegeder, Electronic structure changes during the on-surface synthesis of nitrogen-doped chevron-shaped graphene nanoribbons, Phys. Rev. B 96, 045434 (2017).

[20] P. Jakob (private communication).

[21] See Supplemental Material at http://link.aps.org/supplemental/ 10.1103/PhysRevB.98.075410 for XPS spectra of DBBA after deposition on $\mathrm{Cu}(111)$ and $(3,1)$-GNR after annealing at different temperatures; XPS spectra of 7-AGNR, CGNRs, NCGNRs on $\mathrm{Au}(111)$ and (3,1)-GNR, CGNRs, and NCGNRs on $\mathrm{Cu}(111)$; LEED and ARPES of graphene on $\mathrm{Cu}(111)$; van der Waals radii of $\mathrm{Au}, \mathrm{Cu}, \mathrm{C}$, and $\mathrm{N}$ atoms.

[22] K. A. Simonov, N. A. Vinogradov, A. S. Vinogradov, A. V. Generalov, E. M. Zagrebina, N. Mårtensson, A. A. Cafolla, T. Carpy, J. P. Cunniffe, and A. B. Preobrajenski, Comment on bottom-up graphene-nanoribbon fabrication reveals chiral edges and enantioselectivity, ACS Nano 9, 3399 (2015).

[23] P. Han, K. Akagi, F. Federici Canova, H. Mutoh, S. Shiraki, K. Iwaya, P. S. Weiss, N. Asao, and T. Hitosugi, Reply to comment 
on bottom-up graphene-nanoribbon fabrication reveals chiral edges and enantioselectivity, ACS Nano 9, 3404 (2015).

[24] P. Han, K. Akagi, F. Federici Canova, R. Shimizu, H. Oguchi, S. Shiraki, P. S. Weiss, N. Asao, and T. Hitosugi, Self-assembly strategy for fabricating connected graphene nanoribbons, ACS Nano 9, 12035 (2015).

[25] C. Sanchez-Sanchez, T. Dienel, O. Deniz, P. Ruffieux, R. Berger, X. Feng, K. Müllen, and R. Fasel, Purely armchair or partially chiral: Noncontact atomic force microscopy characterization of dibromo-bianthryl-based graphene nanoribbons grown on cu(111), ACS Nano 10, 8006 (2016).

[26] D. G. de Oteyza, A. García-Lekue, M. Vilas-Varela, N. MerinoDíez, E. Carbonell-Sanromi, M. Corso, G. Vasseur, C. Rogero, E. Guitian, J. I. Pascual, J. E. Ortega, Y. Wakayama, and D. Pena, Substrate-independent growth of atomically precise chiral graphene nanoribbons, ACS Nano 10, 9000 (2016).

[27] F. Schulz, P. H. Jacobse, F. F. Canova, J. van der Lit, D. Z. Gao, A. van den Hoogenband, P. Han, R. J. M. Klein Gebbink, M.-E. Moret, P. M. Joensuu, I. Swart, and P. Liljeroth, Precursor geometry determines the growth mechanism in graphene nanoribbons, J. Phys. Chem. C 121, 2896 (2017).

[28] S. Gottardi, K. Müller, L. Bignardi, J. C. Moreno-López, T. A. Pham, O. Ivashenko, M. Yablonskikh, A. Barinov, J. Björk, P. Rudolf, and M. Stöhr, Comparing graphene growth on $\mathrm{Cu}(111)$ versus oxidized $\mathrm{Cu}(111)$, Nano Lett. 15, 917 (2015).

[29] F. C. Bocquet, G. Mercurio, M. Franke, G. van Straaten, S. Weiß, S. Soubatch, C. Kumpf, and F. S. Tautz, Torricelli: A software to determine atomic spatial distributions from normal incidence x-ray standing wave data, Comput. Phys. Commun. (2018), doi: 10.1016/j.cpc.2018.06.009.

[30] C. J. Fisher, R. Ithin, R. G. Jones, G. J. Jackson, D. P. Woodruff, and B. C. C. Cowie, Non-dipole photoemission effects in X-ray standing wavefield determination of surface structure, J. Phys.: Condens. Matter 10, L623 (1998).

[31] G. van Straaten, M. Franke, F. C. Bocquet, F. S. Tautz, and C. Kumpf, Non-dipolar effects in photoelectron-based normal incidence x-ray standing wave experiments, J. Electron Spectrosc. Relat. Phenom. 222, 106 (2018).

[32] S. Weiß, I. Krieger, T. Heepenstrick, S. Soubatch, M. Sokolowski, and F. S. Tautz, Determination of the adsorption geometry of PTCDA on the $\mathrm{Cu}(100)$ surface, Phys. Rev. B 96, 075414 (2017).

[33] C. Wöll, S. Chiang, R. J. Wilson, and P. H. Lippel, Determination of atom positions at stacking-fault dislocations on $\mathrm{Au}(111)$ by scanning tunneling microscopy, Phys. Rev. B 39, 7988 (1989).

[34] A. R. Sandy, S. G. J. Mochrie, D. M. Zehner, K. G. Huang, and D. Gibbs, Structure and phases of the $\mathrm{Au}(111)$ surface: X-rayscattering measurements, Phys. Rev. B 43, 4667 (1991).

[35] S. ̊. Lindgren, L. Walldén, J. Rundgren, and P. Westrin, Low-energy electron diffraction from $\mathrm{Cu}(111)$ : Subthreshold effect and energy-dependent inner potential; surface relaxation and metric distances between spectra, Phys. Rev. B 29, 576 (1984).

[36] Y. Zhang, Y. Zhang, G. Li, J. Lu, X. Lin, S. Du, R. Berger, X. Feng, K. Müllen, and H.-J. Gao, Direct visualization of atomically precise nitrogen-doped graphene nanoribbons, Appl. Phys. Lett. 105, 023101 (2014).

[37] L. Kilian, A. Hauschild, R. Temirov, S. Soubatch, A. Schöll, A. Bendounan, F. Reinert, T.-L. Lee, F. S. Tautz, M. Sokolowski, and E. Umbach, Role of Intermolecular Interactions on the Electronic and Geometric Structure of A Large $\pi$-Conjugated Molecule Adsorbed on a Metal Surface, Phys. Rev. Lett. 100, 136103 (2008)

[38] M. Willenbockel, R. J. Maurer, C. Bronner, M. Schulze, B. Stadtmüller, S. Soubatch, P. Tegeder, K. Reuter, and F. S. Tautz, Coverage-driven dissociation of azobenzene on $\mathrm{Cu}(111)$ : A route towards defined surface functionalization, Chem. Commun. 51, 15324 (2015).

[39] A. Hauschild, R. Temirov, S. Soubatch, O. Bauer, A. Schöll, B. C. C. Cowie, T.-L. Lee, F. S. Tautz, and M. Sokolowski, Normalincidence $\mathrm{x}$-ray standing-wave determination of the adsorption geometry of PTCDA on $\operatorname{Ag}(111)$ : Comparison of the ordered room-temperature and disordered low-temperature phases, Phys. Rev. B 81, 125432 (2010).

[40] A. Bondi, Van der Waals volumes and radii, J. Phys. Chem. 68, 441 (1964).

[41] M. Willenbockel, D. Lüftner, B. Stadtmüller, G. Koller, C. Kumpf, S. Soubatch, P. Puschnig, M. G. Ramsey, and F. S. Tautz, The interplay between interface structure, energy level alignment and chemical bonding strength at organic-metal interfaces, Phys. Chem. Chem. Phys. 17, 1530 (2015).

[42] P. Ruffieux, J. Cai, N. C. Plumb, L. Patthey, D. Prezzi, A. Ferretti, E. Molinari, X. Feng, K. Müllen, C. A. Pignedoli, and R. Fasel, Electronic structure of atomically precise graphene nanoribbons, ACS Nano 6, 6930 (2012).

[43] C. Bronner, F. Leyssner, S. Stremlau, M. Utecht, P. Saalfrank, T. Klamroth, and P. Tegeder, Electronic structure of a subnanometer wide bottom-up fabricated graphene nanoribbon: End states, band gap, and dispersion, Phys. Rev. B 86, 085444 (2012).

[44] C. Bronner, D. Gerbert, A. Broska, and P. Tegeder, Excitonic states in narrow armchair graphene nanoribbons on gold surfaces, J. Phys. Chem. C 120, 26168 (2016).

[45] David A. Duncan, I09, Diamond Light Source, UK (private communication).

[46] C. Busse, P. Lazić, R. Djemour, J. Coraux, T. Gerber, N. Atodiresei, V. Caciuc, R. Brako, A.T. N’Diaye, S. Blügel, J. Zegenhagen, and T. Michely, Graphene On $\operatorname{Ir}(111)$ : Physisorption with Chemical Modulation, Phys. Rev. Lett. 107, 036101 (2011).

[47] L. Gao, J. R. Guest, and N. P. Guisinger, Epitaxial graphene on $\mathrm{Cu}(111)$, Nano Lett. 10, 3512 (2010).

[48] J. D. Teeter, P. S. Costa, M. Mehdi Pour, D. P. Miller, E. Zurek, A. Enders, and A. Sinitskii, Epitaxial growth of aligned atomically precise chevron graphene nanoribbons on $\mathrm{Cu}(111)$, Chem. Commun. 53, 8463 (2017).

[49] P. Süle, M. Szendrő, C. Hwang, and L. Tapasztó, Rotation misorientated graphene moiré superlattices on $\mathrm{Cu}(111)$ : Classical molecular dynamics simulations and scanning tunneling microscopy studies, Carbon 77, 1082 (2014).

[50] L. Ferrighi, M. I. Trioni, and C. Di Valentin, Boron-doped, nitrogen-doped, and codoped graphene on $\mathrm{Cu}(111)$ : A DFT + vdW study, J. Phys. Chem. C 119, 6056 (2015).

[51] T. Frank, M. Gmitra, and J. Fabian, Theory of electronic and spin-orbit proximity effects in graphene on $\mathrm{Cu}(111)$, Phys. Rev. B 93, 155142 (2016).

[52] H. Ishii, K. Sugiyama, E. Ito, and K. Seki, Energy level alignment and interfacial electronic structures at organic/metal and organic/organic interfaces, Adv. Mater. 11, 605 (1999). 
[53] N. Koch, A. Gerlach, S. Duhm, H. Glowatzki, G. Heimel, A. Vollmer, Y. Sakamoto, T. Suzuki, J. Zegenhagen, J. P. Rabe, and F. Schreiber, Adsorption-induced intramolecular dipole: Correlating molecular conformation and interface electronic structure, J. Am. Chem. Soc. 130, 7300 (2008).
[54] C. Brülke, T. Heepenstrick, N. Humberg, I. Krieger, M. Sokolowski, S. Weiß, F. S. Tautz, and S. Soubatch, Long vertical distance bonding of the hexagonal boron nitride monolayer on the $\mathrm{Cu}(111)$ surface, J. Phys. Chem. C 121, 23964 (2017). 